\title{
Identification and Characterization of Inter-Organizational Information Flows in the Portuguese National Health Service
}

Eduardo Pinto'; António Carvalho Brito'; Ricardo João Cruz-Correia²

${ }^{1}$ INESC TEC, Faculdade de Engenharia, Universidade do Porto;

${ }^{2}$ CINTESIS - Center for Research in Health Technologies and Information Systems, Faculdade de Medicina, Universidade do Porto

\section{Keywords}

eHealth, health information exchange, health information management, health information systems

\section{Summary}

Objectives: To understand and build a collective vision of all existing institutions in the Portuguese National Health Service as well as to perceive how and how far the interaction between those multiple institutions is supported by Information Systems (IS).

Methods: Upon identification of the institutions involved in the healthcare process, a set of interviews with experienced people from those institutions was conducted, which produced about five hours of tape. The research was focused exclusively on processes involving two different organizations and any internal processes were altogether excluded from it.

Results: The study allowed the identification of about 50 recurrent interaction processes, which were classified into four different varieties in accordance with the nature of the information flow: administrative, clinical, identificational and statistical. In addition, these processes were divided in accordance with the way how that integration is achieved, from completely automated to email or telephone-based.

Conclusions: Funds/Money related processes are technologically more rigid and standardized, whereas auditing and inspection ones are less supported by automatic systems. There emerged an interesting level of sharing and integration in clinical processes, although the integration is mostly made at the interface level. The authors identified 5 particularly relevant and dominant actors (2 classes of individuals and 3 institutions) with which there is a need for coordination and cooperation. The authors consider that, in future works, an effort should be made to provide the various institutions with guidelines/interfaces and prompt such institutions to elaborate upon these.

Correspondence to:

Eduardo Pinto

INESC TEC, Campus da FEUP

Rua Dr. Roberto Frias

4200 - 465 Porto, Portugal

Email: eduardo.pinto@fe.up.pt

Funding

The authors would like to thank the Portuguese Ministry of Health and SPMS for the support and FCT for funding this research (SFRH/BD/90838/2012).
Appl Clin Inform 2016; 7: 1202-1220

https://doi.org/10.4338/ACl-2016-08-RA-0135

received: August 3, 2016

accepted: November 5, 2016

published: December 21, 2016

Citation: Pinto E, Brito AC, Cruz-Correia RJ. Identification and characterization of inter-organizational information flows in the Portuguese National Health Service. Appl Clin Inform 2016; 7: 1202-1220 https://doi.org/10.4338/ACl-2016-08-RA-0135 


\section{Background}

All over the world, various countries started programmes, commonly known as Electronic Health Records (EHR), which aim at implementing either partial or complete IT solutions to share clinical information among different institutions, with various degrees of success. Denmark [1,2], England [3], Canada [4-6] or USA [7] are just some of the countries that initiated the complex process of implementing an EHR. These projects are known for their high complexity, cost over budget and frequent problems in achieving the expected results, since they face various technological challenges as well as multiple social and organizational barriers $[8,9]$. Incomplete patient information and insufficient awareness of workflow structure and user roles has been suggested [10] as barriers to the successful implementation of health information exchange projects and the accomplishment of its full value [11].

In Portugal, the Ministry of Health started in 2010 a partnership with the Faculty of Engineering of University of Porto (FEUP) to produce a set of recommendations concerning the design and implementation in Portugal of such a programme. After some years of work and research, a platform called Plataforma de Dados de Saúde (PDS - Health Data Platform) was officially launched in July 2012, the result of the invaluable collaboration between the FEUP and the Serviços Partilhados do Ministério da Saúde (SPMS) - Shared Services of Ministry of Health - the government entity in charge of that process, which the research team was consulted about in three main areas: service design, business processes and information architecture [12-15]. However, this kind of project is in constant evolution and it is unlikely that it might ever be regarded as complete. Hence, in order to continue the previous work, the authors believe it necessary to have a clear and overall knowledge of the Portuguese healthcare framework. Accordingly, we conducted an exploratory study in order to identify and comprehend the role of the various entities involved in the healthcare process as well as the interactions among these.

Knowing that the Portuguese health information systems are afflicted with the same problems [16] that are likely to be found in most countries, we conducted a survey of the available literature on the topic. There is no significant number of papers which specifically identify nationwide information flows or their equivalent. Papers often do not address the problem from a comprehensive perspective, focusing instead on analysis of specific contexts, such as social care services - as in [17], perinatal care - as in [18], psychiatric rehabilitation - as in [19] or drug use - as in [20]. Another research [21] describes how the processes evolved upon implementation of the Health Information Exchange (HIE) technology, drawing a before vs. after comparison within a region of United States, adopting the Information Ecology Framework [22] to structure the analysis. The same framework is also used in [23], in this case to evaluate professionals' experience using a Personal Health Record in the context of Veterans Affairs. From a technological perspective, the exchange of health information can be achieved in multiple ways and be analyzed from at least three perspectives: encoding, transmission and persistence [14]. Thus, a panoply of standards with different pros and cons can be combined [24]. The objective of the present study is to understand and build a collective vision of all existing institutions active in the Portuguese National Health Service as well as to grasp how and how far the interaction between these is supported by Information Systems (IS). Internal systems were not an object of concern, even though these might at times extend their influence to external interactions. We thus aimed at

- understanding the main objectives of each organization;

- eliciting those processes which involve two distinct organizations;

- understanding these interaction processes and the technologies supporting them, and investigating the reasons lying behind the non-implementation of IS support to any given process;

- identifying and classifying the characteristics of the IS used to support inter-organizational processes.

The authors believe this work to be useful to anyone aiming to acquire a comprehensive vision of the Portuguese National Health Service, its institutions and the relations among them, be it a Head of IT Department in an hospital or someone in charge of planning a national-wide project to implement an Electronic Health Record. This research lies in the intersection of two areas of knowledge: the Inter-organizational Information Systems (IOS) and the Electronic Health Records (EHR). Though 
the genesis of this work lies in the EHR challenges, the authors agree that the right approach to these requires a deep understanding of each institution involved in providing healthcare services. This includes institutions with different missions from healthcare service to financing or regulating ones.

The methodologies adopted throughout the research, both to collect the data and to treat and extract information from them are described in section 2, starting with a description of the elicited network of institutions that compose the Portuguese National Health Service. In Section 3 the information flows derived from the interviews are described. In section 4 the discussion of the results is presented and finally in section 5 the conclusions are discussed.

\section{Methods}

Given the paucity of research about information flows at a national level healthcare service and the inherent technologies used by each institution, a qualitative methodology was deemed most appropriate since the goal of this study was to gain an understanding of actors' experiences and perspectives [25]. The study consisted of conduction in-depth interviews with very experienced IT professionals who have direct influence and knowledge about the technologies used in each institution.

\subsection{Data Collection}

The research encompassed two different methods for data collection: preliminary analysis and interviews. The preliminary analysis consisted on identifying the various institutions involved in the Portuguese National Health Service, followed by a work of characterization of the function and purpose of the elicited institutions. The Portuguese National Health Service is constituted by a wide network of several institutions and complex processes aiming to provide a patient with proper healthcare services. We divided the institutions into those which provide healthcare services and the ones which are of a more administrative nature or somehow not focused on healthcare services. $>$ Figure 1 summarizes the organic structure of the various entities identified.

At a second stage, the interviews conducted allowed to gather in-depth information, such as the description of processes, directly from experts' experience. Respondents were selected mainly through a method of judgment sampling and occasionally by snowball sampling [26]. Researchers' previous work in the area allowed selecting persons with a high degree of knowledge and a determinant role in the respective institution. The data collection was conducted until the saturation of relevant interactions was reached, i.e. when new interviews no longer brought new insights [27].

\subsection{Sampling}

Previous studies done in the context of the referred collaboration protocol used healthcare professionals such as doctors and nurses as the source of information to understand their user experience and the difficulties they encounter. Thus, in this study it was relevant to gather a more technical perspective on what was being used to bring the information to the professionals. In that sense, the selected interviewees were experienced professionals in the areas of IS or IT from each one of the institutions. Notes and audio recording were the chosen methods of data collection, and the interview of eight people produced about five hours of tape. The interviewees were as follows:

- Head of Information Systems Unit from the National Authority of Medicines and Healthcare Products (INFARMED)

- Project Manager from the Shared Services of Ministry of Health (SPMS)

- Former IT Consultant from the Central Administration of the Healthcare System (ACSS)

- Head of IT Unit from the Portuguese Institute of Blood and Transplantation (IPST)

- Head of IT Unit from the National Institute of Medical Emergency (INEM)

- Head of Information Systems Unit from a Regional Health Authority (ARS)

- Head of Information Systems Unit from a central Hospital

- Head of Information Systems Unit from a Health Local Unit (ULS) 
The institutions were chosen to comprise different organizational contexts. Thus, the researchers contemplated institutions which provide local healthcare services (such as a ULS or a large hospital), a regional administration institution (ARS) and diverse national organizations (e.g. IPST, INFARMED, etc.).

\subsection{Interviews and Data Analysis}

The interviews were always conducted by the same researcher and averaged 42 minutes. Each interview was divided into 3 parts:

1. a grand tour question aiming at the identification of the organization's main goals;

2. several mini tour questions addressing the existing information flows with other health care related institutions and of the technology that supported these flows or the absence thereof;

3. follow-up mini tour questions towards the grasping of the aforementioned technology.

Responses were both digitally and manually recorded by the researcher. The data collected was reviewed and analyzed throughout the study, which included matching the digital sound recording with the field notes. Any missing notes were added as the notes were converted to a digital format. After theoretical saturation of the data collection was reached, all the interview transcripts were reviewed. For each of the elicited flows, researchers confirmed the existence of a source, a destiny and the form of communication used. Four categories - detailed in this article - were used to classify the flows. Diagrams were found an adequate method to depict and summarize the findings.

Some details were somewhat elusive for several reasons: extreme complexity of systems interaction, whose detailed study would not add to the rigor of the project; systems that despite being used by an institution are not hosted by it, wherefore implementation details are unknown to the said institution; interviewee reluctance in providing sensitive detail.

\section{Results}

In order to better understand the interactions between the different organizations, it was decided to classify each flow within one of the following categories:

- Administrative Flow - information related with processes parallel to healthcare, which includes financial, reporting, accounting or administrative information of another nature;

- Clinical Flow - any information directly related to the clinical condition of a patient (diseases, medication, diagnoses, exams results, etc.)

- Identificational Flow - information that is used to identify patients or health professionals, usually consisting chiefly of demographic information or professional and authentication credentials;

- Statistical Flow - data resulting from the daily activities that later is used by other institutions for research purposes, prevention and control or auditing.

The diagrams included present a general overview on the quantity and nature of the data flows that circulate among the institutions in this ecosystem. The organizations are represented by grey squares and the information flow between any two entities is represented by the arrows connecting them. The line type varies with the flow category as defined above. The diagrams also depict how that information flow happens (as detailed in Table 1).

Finally, the direction of the arrow indicates the source and destination entities of the information flow.

\subsection{Administrative Information Flows}

There are multiple information flows that were considered to be of an administrative nature, which may be handled in several ways. Upon mustering diverse types of interaction, they were grouped as follows (details in $>$ Figure 2): 
- The management of Human Resources (HR) and remunerations: this used to be done internally and reported through the same system that was installed in the several institutions. However, the application has evolved into a web-based version, thus forcing the institutions to adjust their processes to it. This system, provided by SPMS, was later adopted by ACSS, the entity responsible for those processes;

- The regulation of drugs and medical devices available for prescription and other objectives: this process is controlled by INFARMED. Essentially, INFARMED provides the information always in the same way, be it drugs or medical devices, by sharing a Microsoft Access file containing the necessary information. The drugs list, provided daily, is used fundamentally for prescription, dispensation and invoice auditing purposes and the entities that need that list are to incorporate it in their systems. Hospitals, SPMS, ACSS and pharmacies are the most interested entities in this information. The process concerning the medical devices and involving the hospitals and SPMS is identical;

- Production control indicators and services billing by ACSS: this process requires some data from the healthcare providers. On the one hand, the data concerning hospitals is sent directly to ACSS through directly integrated systems. On the other hand, the data related to primary care is first collected by the local ARS and then conveyed to ACSS through an existing UI into a IS;

- Request of blood components: hospitals use a UI for a system maintained by IPST in order to manage the requests related to blood components. Furthermore, INFARMED provides IPST with information about defects in medical devices such as blood collection bags by email.

\subsection{Clinical Information Flows}

The flows elicited that contain clinical data directly related to a patient were gathered as follows (details in $>$ Figure 3):

- Drugs prescription: physicians have recently been made to adopt a new web-based drugs prescription system. That IS is provided by SPMS and is used in both hospitals and primary care units;

- Emergency-related processes: INEM - the entity responsible for providing emergency healthcare services - also transports the victim to a hospital. In that process, information about the clinical condition of the victim is sent by email to the hospital that is to receive them. Later, the emergency related entries are made available for consultation by a physician through an application provided by SPMS that fetches those entries from INEM (by a web-service). Furthermore, a DGS support hotline named Linha de Saúde24 - (Health Line 24) is available, that answers patients doubts about their symptomatology and redirects them to a nearby hospital when appropriate, in which case the information about that patient is immediately conveyed to the hospital by email;

- Medical specialty referrals: ACSS is the entity responsible for the system that allows physicians to refer patients to specialist doctors. The IS used by physicians to input these referrals tries to balance all requests taking their urgency into account;

- Screenings: each ARS uses an IS to gather information about the screenings that are run in its jurisdiction;

- Electronic Medical Records (EMR): currently, it is already possible for physicians to access the EMR of a patient from another hospital or primary-care unit. That process is supported by PDS (Plataforma de Dados da Sáude - Healthcare Data Platform) and allows the physicians to access such information through an interface provided by the institution that holds these data. This flow occurs frequently from primary-care units to hospitals or from hospitals to other hospitals. In addition, some hospitals - usually geographically close to each other - develop integration systems between their PACS (Picture Archiving and Communication System) so that they are able to share imaging exams. Hospitals tend to develop peer integrations with service providers, to allow for instance exam results to be automatically integrated into their systems;

- Telemedicine: some hospitals need to contract external services such as a specialist's opinion on a determined patient. Thus, they need to send the patient's data to the physician and that process is usually done by email. There are also some flows to support teleradiology, between a hospital or ULS and a service provider. In this case, the adoption of a web-service allows the process to be more automatic than when using email. 


\subsection{Identificational Information Flows}

As we will describe, the flows considered to be about identification processes are predominantly supported by ISs or their direct integration. The main processes are described as follows ( $\triangleright$ Figure 4):

- Identification and authentication of professionals: the professional associations are the competent entities to identify the professionals at a national level. The information flows between those entities and SPMS are supported by a web-service provided by the Dentists Association and the Medical Association so as to allow the SPMS to confirm the identity and professional credentials of the professionals in their systems. Furthermore, a similar process is adopted to allow INFARMED to identify the pharmacists. Lastly, DGS provides the SPMS with the identification of the physicians that collaborate in audits and supervision processes;

- Registration of deaths: the hospitals use an IS provided by SPMS to introduce information about patient death. In addition, another IS provided by SPMS is used by hospitals to manage the healthcare services that are supported by insurance companies;

- Donor exemptions: donors benefit from some exemptions in terms of healthcare services cost, wherefore data about donors who benefit from some exemption is sent from IPST to ACSS, so that the latter is informed about the people who benefit from that kind of privilege;

- Information regarding prescribed exams: some hospitals develop direct integration systems with service providers, such as private clinics for medical analysis and laboratories, so as to automatically send that provider demographic information concerning the patients and to have the results sent back later.

\subsection{Statistical Information Flows}

The statistical data flows elicited were divided into three groups (details in $>$ Figure 5):

- Clinical procedures, diagnosis and the like, used as input for auditing processes by institutions such as ERS, IGAS and DGS. This information is required by the auditors, commonly for a form filling processes using Microsoft Excel files;

- Resource consumption, such as the reporting regarding the consumption of medical supplies made by hospitals to INFARMED, usually submitted by manual processes like email, or the blood reserves availability reports to IPST sent automatically by a service running in the hospital;

- Public health information such as the indicators that are sent from INEM and ARS to DGS - information sent by email or acquired by using an UI to consult it. Another related report is submitted by hospitals to IPST when reporting the adverse reactions caused by tissue transplants and related procedures, using a website provided by IPST.

\subsection{Mapping the Information Ecology Concepts}

The environment described is highly complex, involving multiple actors and interactions among these. This diversity implies permanent evolving relations between people, institutions and technology. About 50 interactions were elicited and classified in terms of their process context and automation level. In order to better understand and structure the context, the researchers have applied the Information Ecology Framework [22]. Said concept has been previously proved useful [28] and applied by other authors to depict a healthcare context [23]. The mapping between the framework and the PNHS is done hereinafter.

The Information Ecology Framework uses an approach both social and technical to understand, describe and characterize relations between people and technology in a specific context. Based on the interviews and information gathered, the authors mapped the five main components to the Portuguese National Health Service context as follows.

\section{Ecology Component: System}

As described, the Portuguese National Health Service is an environment constituted by several different organizations, each with its own roles and objectives. From auditing to healthcare organizations, the volume of data flow is very significant and includes not only clinical information but also 
financial or statistical data that are directly related to healthcare. As described, information exchange serves multiple purposes such as continuity of care - including transfers between inpatient and outpatient environments, and the fostering of service response and improvement awareness.

\section{Ecology Component: Locality}

The identification and characterization of the several institutions that compose the National Health Service aimed to fully capture this context. As mentioned, some software is developed centrally and used in various institutions. Yet, institutions such as hospitals usually have their own technical departments which adjust, customize and integrate these applications into their internal software. This fact, along with the existence of five regional health authorities across the country, highlights the existence of a strong regional consciousness.

\section{Ecology Component: Diversity}

Although not explicitly identified, the information flows are brought about by the action of individuals who are performing their role within the system, from patients to physicians, from administrative staff to nurses. Most of them trigger information exchanges in multiple ways by playing a variety of roles in the system. Patients are the main driver: when they move between clinical sites, nurses and physicians seek information which allows them to increase the service quality and effectiveness - information that is often external and needs to be transferred from another healthcare institution.

\section{Ecology Component: Keystone Species}

The keystone species are described as "species whose presence is crucial to the survival of the ecology itself". In this context, we identified two types of keystone species:

1. Individuals: namely patients, at the center of the healthcare services and the most important element in the ecosystem, and healthcare professionals.. The patient is the common element be it in ambulatory, in the emergencies, in primary-care units, pharmacies, and the like. The patient is the primary promoter of the share of clinical information even if indirectly, as is for instance the case of financial and statistical data reported from hospitals to the central entities. On the other hand, the caregivers, such as physicians, nurses, psychologists and the like, are a critical force on the side of the institutions; they are essential to provide each patient with the necessary care, as well as to assure the continuity of this care, both from service to service and institution to institution.

2. Institutions: as Keystone Species, we would like to refer the institutions with the highest number of information exchange flows, namely the Shared Services of the Ministry of Health (SPMS) with 15, the Regional Health Authorities (ARS) with 14 and hospitals with 22. From the standpoint of clinical information sharing, to ensure the continuity of care across the diverse services and institutions, two of these play a major role: SPMS and ARS. The first (SPMS) is responsible for a significant share of the software used across the whole ecosystem, from development to deployment, while the second (ARS) places a special emphasis on the regulation of regional services concerning mainly the primary-care units, which are usually very resource-dependent on the superordinate ARS. Lastly, hospitals are constituted by different units and departments, whereby their IT departments often have to deal with inter-departmental data integration, not to mention the growing need for information exchange with other institutions, such as other hospitals, primary-care units, emergency agencies and the like.

\section{Ecology Component: Coevolution}

As described in the Information Ecology Framework, the ecology implies the existence of a dynamic environment that evolves over time. In the context of the Portuguese National Health Service - and for that matter in the context of any National Health Service - the following is certainly to be expected:

1. Shifting processes: owing to the inherent evolution of traditional processes, and the increasing dependence on technology;

2. Evolving services: factors like economy, finance, demography, politics or technology significantly affect the services that are provide by each healthcare organization. The services and the institu- 
tions themselves are dynamic elements in the system, often forced to adapt to the surrounding environment;

3. New players: the dynamic of the healthcare services is also favorable to the emergence of new players as e.g. new public or private institutions, as well as of new software solutions or innovative services developed by software houses or by the ministry.

\section{Discussion}

\subsection{Entities Mission vs Flows}

We can start by trying to make some remarks about how the mission of some organizations fits the type of flow that they generally use.

For instance, INFARMED stands out as a very relevant source of information for several other institutions, providing catalogues of available drugs and medical devices. Although the information sharing process has been automated and actually works, it is based on the sharing of Microsoft Access files, a most uncommon solution for this kind of problem. The retrieval through a service request of data in XML or some other format is likely to be advisable.

The interactions with the professional associations relate mainly to the identification of professionals. Although the contribution of these entities exceeds the professionals identification, these were the interactions that were found to be IS supported and to occur in a more regular and automated basis. It is worthy of note that there is no information flow regarding the identification of nurses. This means that nurses are always identified locally with all the limitations that can be inferred. Regarding this, it is important to refer that in country wide identity management, the existence of different regions or simply of multiple institutions is a recurrent challenge for EHR implementations and has been the subject of some interesting discussions [29]. A European Commission's report states that "a key component of any national or regional eHealth infrastructure is the ability to uniquely identify electronically citizens/patients, healthcare professionals, healthcare providers, and pharmacies" [30].

In what regards the analysis of healthcare providers, hospitals are authentic hubs of processes and information. Even without considering internal processes, the need for information and the interactions that hospitals are supposed to support certainly bring several challenges and issues to their IT departments. Although there apparently begin to appear some directives from the Ministry of Health in terms of norms and standards, such as a national Catalogue of Allergies and Other Adverse Reactions or a national Catalogue of Medical Devices, the systems implemented to date that are working were generally driven by a judgment based on local specificities and constraints and lack a national strategic vision.

\subsection{Different Technological Processes}

In general terms, the various levels of integration achieved appeared to be independent of the information flow type. Nevertheless, some tendencies depending on the flow context and data are discernible.

In terms of administrative flows ( $\triangleright$ Figure 2), it is noticeable that all financial processes (e.g. HR \& salaries, billing, production indicators) are mostly computerized, either by direct integration with the ACSS systems or mainly by using an IS provided to introduce information. Conversely, the process used by INFARMED to distribute catalogues is the sharing of a Microsoft Access file.

The clinical flows ( Figure 3) are generally supported by an IS user interfaces. That is to say, rather than transferring the information directly from one system to another, a common solution is to grant access to the IS with the information. For instance, for a physician to retrieve an EMR of a patient from another institution, access to an UI of the relevant IS is granted to that physician. This process is mediated by a system provided by the SPMS (PDS) that ensures the access of the user to the target IS interface.

Regarding the information flows used to identify professionals or patients, $>$ Figure 4 shows that the SPMS plays a crucial role. Most of these processes occur directly between systems using web-ser- 
vices. For instance, SPMS acts as the broker whenever a communication process between two entities requests an identity confirmation be it of a patient or of a physician.

The information that is deemed to be of a statistical nature ( $>$ Figure 5 ) was found to be seldom automated. In these cases, it often happens that the information exchange takes place by email after filling out a given Excel form. In fact, the Microsoft Excel is a very popular tool for this kind of information flow, as frequently mentioned above. Actually, email is the most commonly used tool to convey information.

\subsection{Common Approach}

One common approach that we have been able to identify is the development and provision of a UI to grant access to some information that someone else uses. This kind of thinking leads to an UI proliferation and consequently to the greater effort of a user, who is obligated to use each one of them when performing their daily tasks. One interviewee referred the most common complaints: the supervisory entity imposed the use of a system and forced the collaborators to use it. However, that IS was unable to meet the functional needs of the supervised entity, which resulted in the use of two different ISs with overlapping functionalities. In fact, user acceptance and willingness to change is often pointed out as one of the main challenges in, for instance, an EHR implementation. And when they do not believe the system befits the purpose, "they tend to employ workarounds which are often unanticipated by management" [31].

Another common issue: hospitals contract geographically close service providers whenever they do not have enough resources, either human or technical, to achieve the levels of productivity imposed upon them. In these cases, peer-to-peer solutions to support some processes between the service providers and the hospital are frequently developed. These peer-to-peer solutions are not the problem, which, as referred above, is all about the absence of national guidelines to perform this kind of flow. This lack of guidelines leads to a proliferation of solutions, the convergence of which, some point out, will be very hard to achieve in the future.

That said, we must add that the existence of international norms and recommendations [24,32] has been a reality for several years (e.g. HL7, IHE, etc.). In fact, when talking specifically about clinical data, generally neither the information is properly coded nor the conveying process is standardized, which means that the central entities were not the only ones responsible for not ensuring the adoption of standards. The local organizations were never too keen on standardized integration, opting instead for expeditious solutions that solve immediate problems to the detriment of longterm ones.

\subsection{Other systems in the world}

The direct comparison with other countries is not straightforward since we were not able to find other country-level organization-based analysis in the literature. Nevertheless, one can find touchpoints with some international case studies.

Unertl et al. [21] analyzed the impact of health information exchange in a region of United Sates using the Information Ecology Framework. Although the two analysis are done from different levels (regional level vs. national level) some resemblances and contrasts can be drawn out between the mentioned study and the present work. Both studies argue for a strong sense of locality as both contexts comprise multiple institutions and individual sites within organizations as well as clearly identify the diversity in both systems. On the other hand, information consumers, exchange facilitators and reservoirs are the three keystone species identify by the mentioned study. Despite the differences, in either ecosystems healthcare professionals are regarded as a keystone specie acting both as an information consumer and as an informal information reservoir. Another keystone specie elicited are the information exchange facilitators even though in the mentioned study these are people and in our study these are the organizations with a higher number of flows.It is worth noting the study from Currie and Suhomlinova [33] that describes the power of "institutional forces upon knowledge sharing" in the United Kingdom National Health Service. Although the mentioned study is done from a professionals' perspective rather than organizations' perspective, all the organizations 
in this context are composed of people and as we referred previously both patients and professionals play a major role in every health information exchange system.

In Denmark, the EHR implementation process started with the definition of six principal information flows with the general practitioner at the center of all the process [34]. The definition of precise standards, a gradual approach or financial incentives are some of the reasons appointed for the success of their project. Regardless of our study being broader than only clinical information flows, the Danish example shows how important of a role SPMS can play as an entity that manages a large majority of the information flows in the Portuguese Health Service and that is able to drive these information flows into more standardized exchanges.

\section{Conclusions}

In this study, the authors were able to depict a national-level perspective on the organization of Portuguese National Healthcare Service by identifying the existing information flows between the different institutions. Additionally, the identified flows were classified by their type both in terms of the data being transferred as well as the integration level they are done at.

In general terms, funds/money related processes tend to be more automated, rigid and standardized, while auditing and inspection processes are prone to be less supported by information systems, what is understandable given not only their less regular nature but also the need for independence of the entities who perform such work. Not all processes need to be completely automated and computerized nor would such level of automation make necessarily sense. Some flows were hard to classify since the same information can be used for different purposes, depending on context. Although we do not address this issue in this work, another common difficulty is grasping where the data is physically stored. Such difficulty arises from the fact that a number of entities are often involved in the process of development and deployment of a given system, i.e. a supervisory entity, a development software house, hospitals or healthcare providers, etc.

In terms of clinical processes, there starts to be an interesting level of sharing and integration. However, the integration is mostly made at a presentation level, which means that the users are forced to jump between applications instead of being able to smoothly access the required information, regardless of where it needs to be retrieved from. Although these integrations at a presentation level can bring short-term advantages, the long-term vision requires changes at other levels. There have been some developments in terms of clarifying at a national level what norms and catalogues should be used (e.g. the Catalogue of Allergies and Other Adverse Reactions, the Catalogue of Medical Devices and the electronic medical discharge letter) and we trust these to be steps in the right direction. Moreover, we believe that the adoption of the new norms should be fostered in the development of new projects such as PDS, thus serving as a catalyst for the other institutions. However, the process will be naturally slow since it involves the modification of long-established processes.

The mapping of the Portuguese context as an information ecology scenario allowed the clarification of the importance of some aspects. The identification of the keystone species is especially relevant when developing and deploying projects that affect the balance of the system. In that regard, we saw that patients and healthcare professionals on one hand and SPMS, hospitals and ARSs on the other hand are the heavy players. Therefore, a successful solution will necessarily depend significantly on these actors.

\section{Future Work}

We believe that one way to improve the pace of clinical information sharing is to distribute the effort as much as possible among the various parties involved. In that sense, SPMS would have a main role by providing the guidelines and urging the several institutions to make their own developments. The policies of imposing more and more systems have proven not to be so successful, particularly in the case of clinical-related systems. We believe that new models such as Metropolis Model [35] - that suggest some principles to generate value in highly crowded contexts - can befit the healthcare services area and be a good asset in order to increase inter-organizational processes efficiency. In future 
research, we intend to address these possibilities, in an attempt to comprehend how to apply those crowdsource-based models to a sector which continuous to struggle to integrate technology into the daily practices and processes.

\section{Clinical Relevance Statement}

Our study allows for a large-scale observation of the existing interconnected health information systems and institutions using information ecology to identify two keystone species: both patients and clinical providers and a central entity for shared services.

\section{Conflicts of Interest}

The authors declare that they have no conflicts of interest in the research.

\section{Human Subjects Protections}

The study was performed in compliance with the World Medical Association Declaration of Helsinki on Ethical Principles for Medical Research Involving Human Subjects and informed consent was obtained in all cases. 


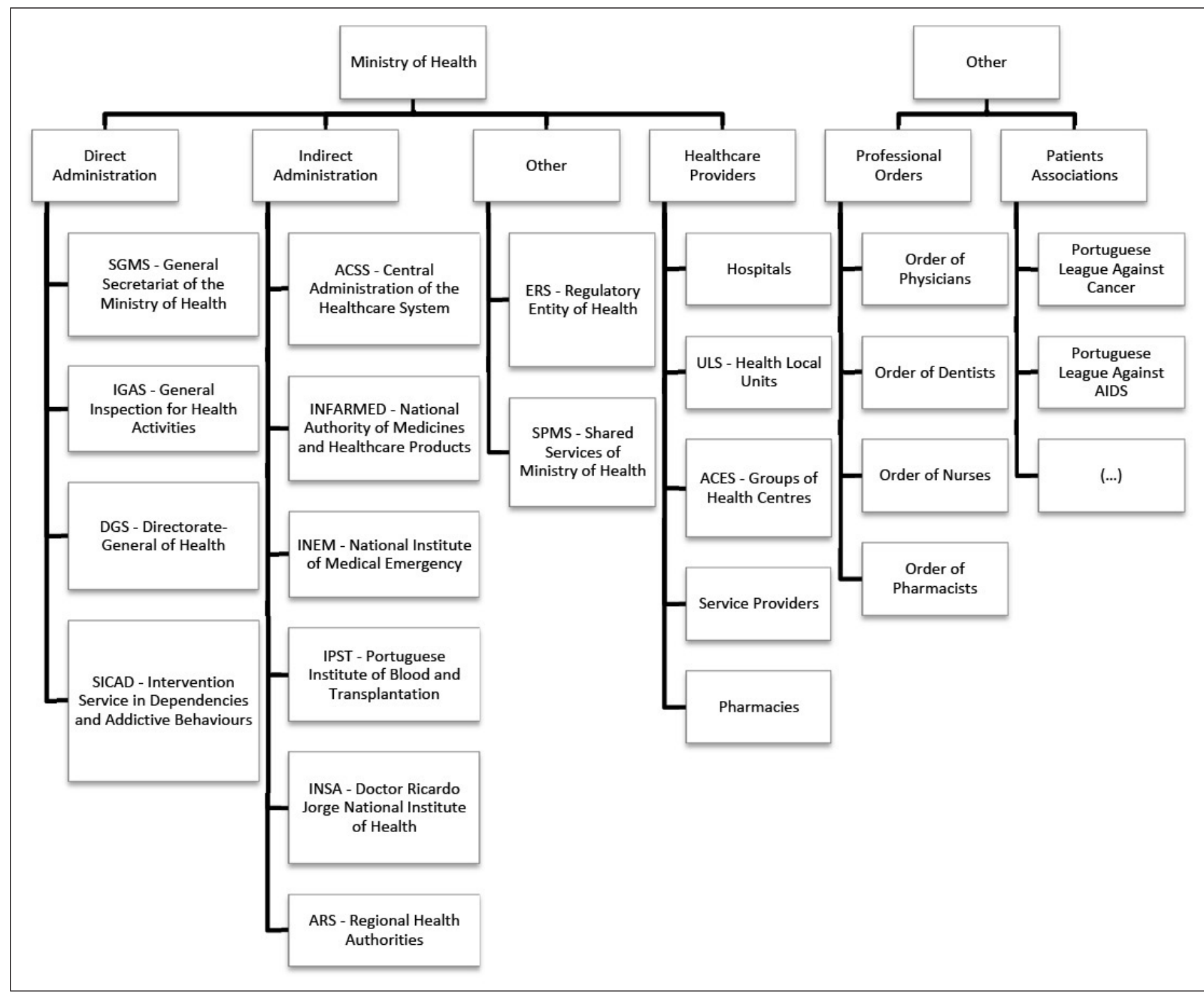

Fig. 1 Organic structure of the Portuguese National Health Service. As expected, the Ministry of Health plays a relevant role but delegates several specific competences in other entities that are under direct or indirect supervision. Most of those are entities with national scope (except the ARS) that are required to develop initiatives or manage a certain area (e.g. INFARMED, INEM, etc.). Other relevant entities are the professional orders on one way and the Patient Associations on the other. The first usually works on regulating the profession and the second providing assistance and disseminating information with patients with a specific disease. 


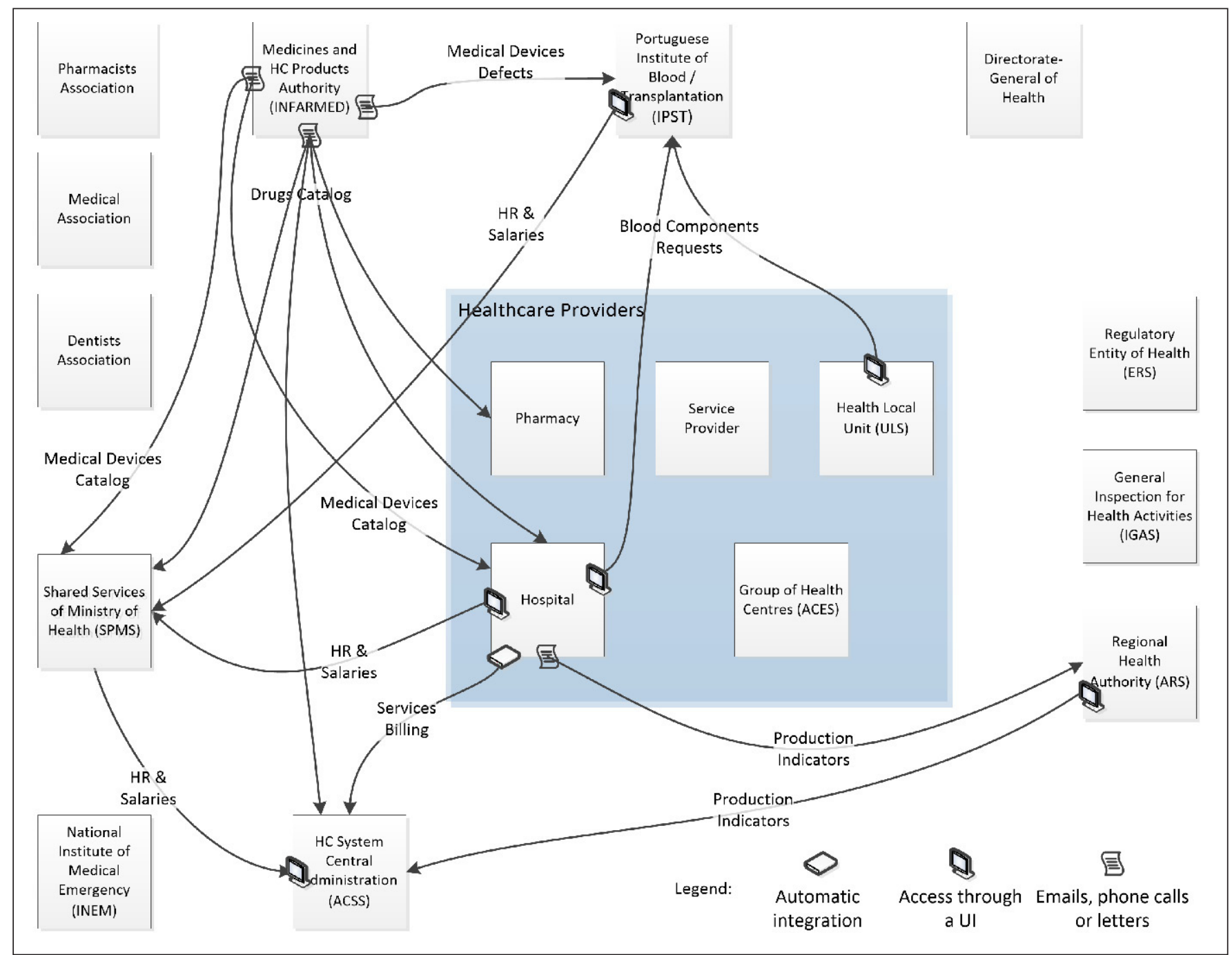

Fig. 2 Representation of administrative information flows elicited from the interviews in which INFARMED has a special preponderance. 


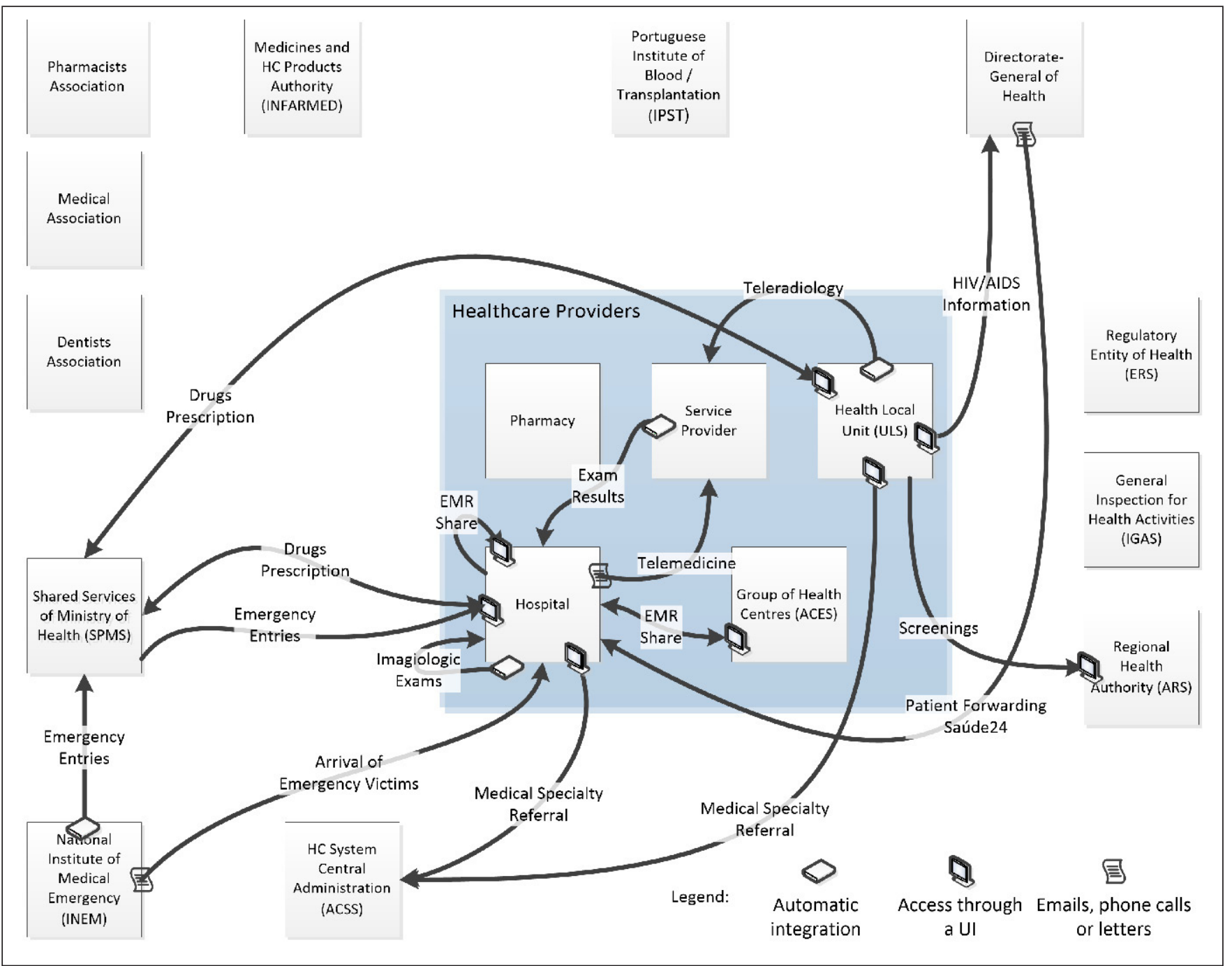

Fig. 3 Representation of clinical information flows elicited from the interviews. As expected, the healthcare providers have a special preponderance. 


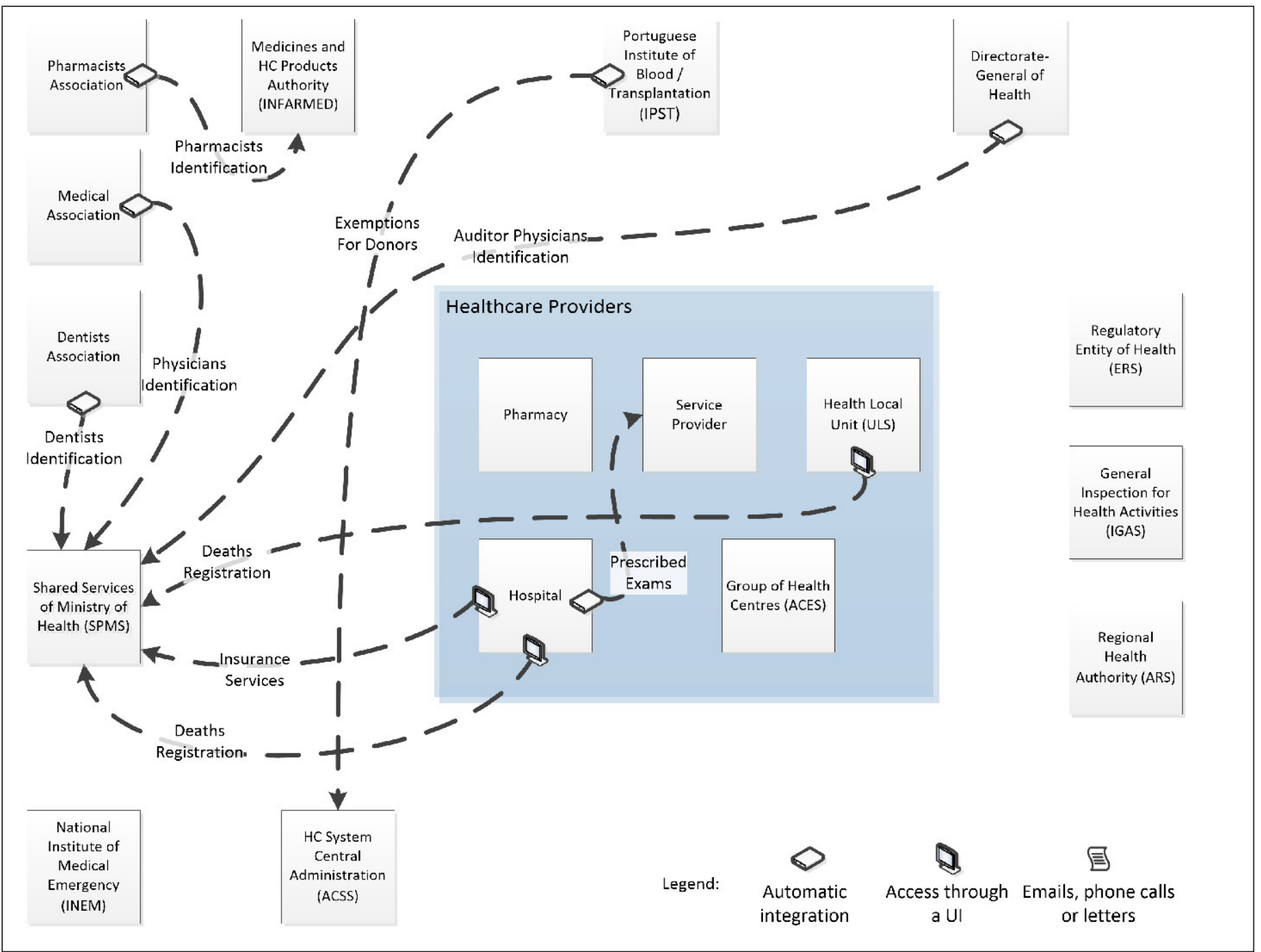

Fig. 4 Representation of information flows that allow identification of professionals or patients usually based on demographic data and in which SPMS plays a significant role. 


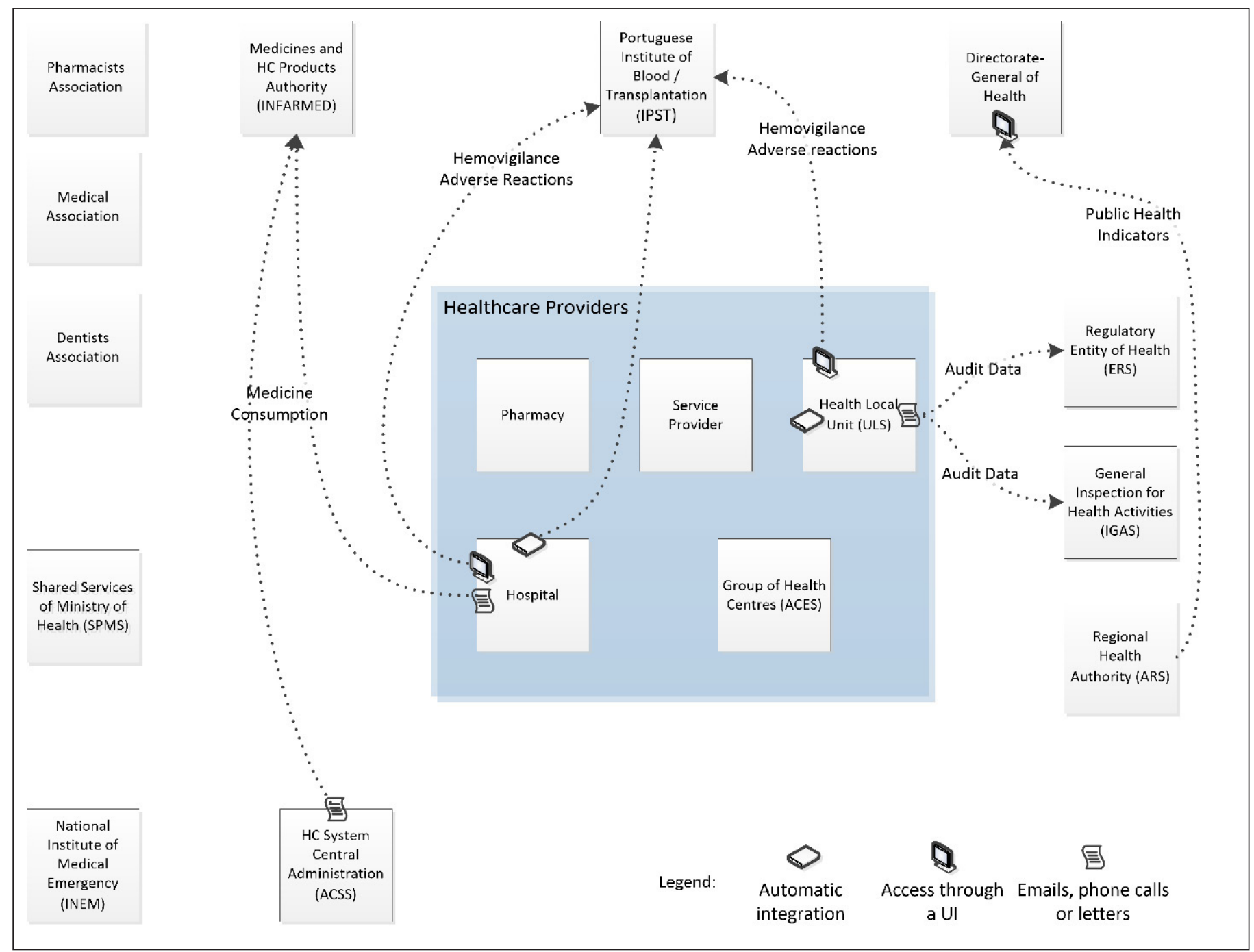

Fig. 5 Representation of statistical information flows that are generally related with audit data or public health concerns. 
Table 1 Legend of the icons used in the schemes to represent the information flows.

Image
automatic integration between systems - represented by a modem image which also repre-
sents the entity that possesses the data
someone accesses a system provided by the other entity (usually web-based) - represented
exchange of emails, phone calls or letters - represented by a paper image in the source of the
connection




\section{References}

1. Bernstein K, Bruun-Rasmussen M, Vingtoft S, Andersen SK, Nøhr C. Modelling and implementing electronic health records in Denmark. Int J Med Inform 2005; 74(2-4): 213-220.

2. Protti D, Johansen I, Perez-Torres F. Comparing the application of Health Information Technology in primary care in Denmark and Andalucía, Spain. Int J Med Inform 2009; 78(4): 270-283.

3. Brennan S. The biggest computer programme in the world ever! How's it going? J Inf Technol 2007; 22(3): 202-211.

4. McGinn CA, Gagnon M-P, Shaw N, Sicotte C, Mathieu L, Leduc Y, Grenier S, Duplantie J, Abdeljelil A Ben, Légaré F. Users' perspectives of key factors to implementing electronic health records in Canada: a Delphi study. BMC Med Inform Decis Mak 2012; 12: 105.

5. Urowitz S, Wiljer D, Apatu E, Eysenbach G, Delenardo C, Harth T, Pai H, Leonard KJ. Is Canada ready for patient accessible electronic health records? A national scan. BMC Med Inform Decis Mak 2008; 8: 33.

6. Rozenblum R, Jang Y, Zimlichman E, Salzberg C, Tamblyn M, Buckeridge D, Forster A, Bates DW, Tamblyn R. A qualitative study of Canada's experience with the implementation of electronic health information technology. C Can Med Assoc J 2011; 183(5): 281-288.

7. Grabenbauer L, Fraser R, McClay J, Woelfl N, Thompson CB, Cambell J, Windle J. Adoption of electronic health records: A qualitative study of academic and private physicians and health administrators. Appl Clin Inform 2011; 2(2): 165-176.

8. Anderson JG. Social, ethical and legal barriers to e-health. Int J Med Inform 2006; 76(5-6): 480-483.

9. Grabenbauer L, Skinner A, Windle J. Electronic Health Record Adoption - Maybe It's not about the Money: Physician Super-Users, Electronic Health Records and Patient Care. Appl Clin Inform 2011; 2(4): 460-471.

10.Eden KB, Totten AM, Kassakian SZ, Gorman PN, McDonagh MS, Devine B, Pappas M, Daeges M, Woods S, Hersh WR. Barriers and facilitators to exchanging health information: A systematic review. Int J Med Inform 2016; 88: 44-51.

11. Walker J, Pan E, Johnston D, Adler-Milstein J, Bates DW, Middleton B. The value of health care information exchange and interoperability. Health Aff (Millwood). 2005; Suppl Web.

12.Pinho NF de, Beirão G, Patrício L, Fisk RP. Understanding value co-creation in complex services with many actors. J Serv Manag 2014; 25(4): 470-493.

13.Patrício L, Brito AC, Pinho N, Oliveira M, Pinto E. Designing a sustainable Electronic Health Record区: from Service Ecosystem to Information Architecture. 2012. Available from: http://paginas.fe.up.pt/ ehisa/ presentations/LiaPatricio_AntonioBrito_PDS_FEUP.pdf

14. Pinto E, Brito AC. Architectural Key Dimensions for a Successful Electronic Health Record Implementation. In: ICEIS 2014 - 16th International Conference on Enterprise Information Systems 2014: 136-143.

15. Pinto E, Brito AC. The Portuguese Health Data Platform: An Alternative Architecture Approach. In: 8th International Conference on e-Health. 2016.

16.Deloitte. Saúde em análise - Uma visão para o futuro. 2011.

17. Richardson S. Inter-agency Information Sharing in Health and Social Care Services: The Role of Professional Culture. Br J Soc Work 2005; 36(4): 657-669.

18. Pieters A. Care and cure: Compete or collaborate? Improving inter-organizational designs in healthcare. A case study in Dutch perinatal care 2013.

19. Timpka T, Bång M, Delbanco T, Walker J. Information infrastructure for inter-organizational mental health services: an actor network theory analysis of psychiatric rehabilitation. J Biomed Inform 2007; 40(4): 429-437.

20.Pouloudi A, Whitley E. Stakeholder identification in inter-organizational systems: gaining insights for drug use management systems. Eur J Inf Syst 1997; 6(1): 1-14.

21. Unertl KM, Johnson KB, Gadd CS, Lorenzi NM. Bridging Organizational Divides in Health Care: An Ecological View of Health Information Exchange. JMIR Med Informatics 2013; 1(1): e3.

22. Nardi BA, O’Day V. Information ecologies: Using technologies with heart. First Monday 1999; 4(5): 1-5.

23. Nazi KM. The personal health record paradox: health care professionals' perspectives and the information ecology of personal health record systems in organizational and clinical settings. J Med Internet Res 2013; 15(4).

24. Atalag K, Kingsford D, Paton C, Warren J. Putting Health Record Interoperability Standards to Work. Electron J Heal Informatics 2010; 5(1): 1-17.

25.Strauss A, Corbin J. Basics of Qualitative Research: Techniques and Procedures for Developing Grounded Theory. Vol. 3, Sage Publications Inc. 2008: 379.

26. Margaret C. Harrell; Melissa A. Bradley. Data Collection Methods Semi-Structured Interviews and Focus Groups. CA: RAND Corporation 2009: 148. 
27. Charmaz K. Constructing grounded theory: a practical guide through qualitative analysis Sage. SAGE; 2006.

28. Fedorowicz J, Gogan J, Ray A. The Ecology of Inter-organizational Information Sharing. J Int Inf Manag 2004; 13(2): 73-86.

29. Campos M joão, Correia M, Antunes L. Leveraging identity management interoperability in ehealth. IEEE Conf Secur Technol ICCST 2011.

30.Stroetmann KA, Artmann J, Stroetmann VN. European countries on their journey towards national eHealth infrastructures. eHealth Strategies 2011.

31. Cresswell KM, Worth A, Sheikh A. Integration of a nationally procured electronic health record system into user work practices. BMC Med Inform Decis Mak 2012; 12(1): 15.

32.Eichelberg M, Aden T, Riesmeier J, Dogac A, Laleci GB. A survey and analysis of Electronic Healthcare Record standards. ACM Comput Surv 2005; 37(4): 277-315.

33. Currie G, Suhomlinova O. The Impact of Institutional Forces Upon Knowledge Sharing in the UK NHS: The Triumph of Professional Power and the Inconsistency of Policy. Public Adm 2006; 84(1): 1-30.

34.Edwards J. Case Study区: Denmark's Achievements With Healthcare Information Exchange. Gart Ind Res Publ 2006.

35.Kazman R, Chen H-M. The metropolis model a new logic for development of crowdsourced systems. Commun ACM 2009; 52(7): 76. 